\title{
Higher Sensitivity of Human Auditory Nerve Fibers to Positive Electrical Currents
}

\author{
Olivier Macherey ${ }^{1,2}$, Robert P. Carlyon ${ }^{2}$, Astrid van Wieringen ${ }^{1}$, John M. Deeks ${ }^{2}$, and Jan Wouters ${ }^{1}$ \\ ${ }^{1}$ ExpORL, Department of Neurosciences, Katholieke Universiteit Leuven, O. E N N2, Herestraat 49 bus 721, 3000 Leuven, Belgium \\ ${ }^{2}$ MRC Cognition and Brain Sciences Unit, 15 Chaucer Road, Cambridge CB2 7EF, UK
}

Received: 8 October 2007; Accepted: 16 January 2008; Online publication: 21 February 2008

\begin{abstract}
Most contemporary cochlear implants (CIs) stimulate the auditory nerve with trains of amplitude-modulated, symmetric biphasic pulses. Although both polarities of a pulse can depolarize the nerve fibers and generate action potentials, it remains unknown which of the two (positive or negative) phases has the stronger effect. Understanding the effects of pulse polarity will help to optimize the stimulation protocols and to deliver the most relevant information to the implant listeners. Animal experiments have shown that cathodic (negative) current flows are more effective than anodic (positive) ones in eliciting neural responses, and this finding has motivated the development of novel speech-processing algorithms. In this study, we show electrophysiologically and psychophysically that the human auditory system exhibits the opposite pattern, being more sensitive to anodic stimulation. We measured electrically evoked compound action potentials in CI listeners for phase-separated pulses, allowing us to tease out the responses to each of the two oppositepolarity phases. At an equal stimulus level, the anodic phase yielded the larger response. Furthermore, a measure of psychophysical masking patterns revealed that this polarity difference was still present at higher levels of the auditory system and was therefore not solely due to antidromic propagation of the neural response. This finding may relate to a particular orientation of the nerve fibers relative to the electrode or to a substantial degeneration and demyelination of
\end{abstract}

Correspondence to: Olivier Macherey - MRC Cognition and Brain Sciences Unit - 15 Chaucer Road, Cambridge CB2 7EF, UK. Telephone: +44 (0) 1223 355294; fax: +44 (0) 1223 359062; email: olivier.macherey@mrc-cbu.cam.ac.uk the peripheral processes. Potential applications to improve CI speech-processing strategies are discussed.

Keywords: cochlear implants, electrical stimulation, auditory nerve, psychophysical masking, stimulus polarity, asymmetric pulses

\section{INTRODUCTION}

Polarity sensitivity of auditory nerve fibers has been extensively studied in animal and computational models using both monophasic and biphasic stimuli. Responses to monophasic cathodic pulses show, in general, lower thresholds and longer latencies than anodic responses (Miller et al. 1998, 1999a, 2004). Similarly, the most effective phase of sinusoidal stimuli was shown to be the cathodic phase in single-fiber recordings from the cat (Hartmann et al. 1984). However, polarity sensitivity may also depend on the electrode position relative to the fibers and on the nerve morphology (Ranck 1975; McIntyre and Grill 1999; Rubinstein 1993; Rattay 1999; Rattay et al. 2001a). If so, then, the findings obtained in the vast majority of animal experiments may not apply to human cochlear implant (CI) listeners (Miller et al. 1998).

In human CI stimulation, charge-balanced stimuli are required to prevent damaging electrochemical reactions (Brummer and Turner 1977). Consequently, the effect of absolute polarity, which is usually studied using monophasic pulses, remains largely unknown in humans. Asymmetric pulses, where the second phase is lower in amplitude and longer in duration than the first, have provided a way to study polarity sensitivity in CI users. In such pulses, the contribution of the short, high-amplitude phase is increased at the expense of 
the long, low-amplitude one while maintaining the charge-balancing requirement. We recently showed that inverting the polarity of an asymmetric pulse stimulus yielded differences in most comfortable levels (MCLs) of CI users, giving lower MCLs when the short, high-amplitude phase was anodic than when it was cathodic (Macherey et al. 2006). Because short pulses require less charge to evoke the same loudness as longer ones (at least for phase durations less than approximately $500 \mu \mathrm{s}$; Moon et al. 1993; Macherey et al. 2006), it is possible that loudness was determined primarily by the higher-amplitude phase of our asymmetric pulses. This result, then, suggests that the human auditory nerve might be more sensitive to anodic stimulation - the opposite to what one expects from animal measures of auditory-nerve activity (Hartmann et al. 1984; Miller et al. 1998, 1999a, 2004). The following series of experiments directly test this hypothesis in eight CI users, using both electrophysiological and psychophysical measures. The experimental design relies on the characteristics of the auditory nerve fibers which, after a stimulation pulse, undergo a period of absolute refractoriness, lasting approximately $400 \mu \mathrm{s}$, followed by a progressive recovery (Stypulkowski and van den Honert 1984; Miller et al. 2001; Morsnowski et al. 2006). Experiment 1 provides an insight into the morphology of anodic and cathodic phase responses. Experiments 2 and 3 measure the masking effectiveness of each individual polarity phase using electrophysiological and psychophysical masking, respectively.

\section{GENERAL METHODS}

\section{Subjects}

Eight users of the CII or HiRes 90k CIs, manufactured by Advanced Bionics, participated in this series of electrophysiological (experiment 1 and 2) and psychophysical (experiment 3) experiments. The subjects' biographical data are shown in Table 1 . Testing was approved by the Cambridge and Leuven Local

TABLE 1

Biographical data of the subjects, including age, duration of deafness (DD) and duration of $\mathrm{Cl}$ use $(\mathrm{Cl})$, in years and etiology

\begin{tabular}{lcrrl}
\hline Subject & Age (years) & $D D$ (years) & $C l$ (years) & \multicolumn{1}{c}{ Etiology } \\
\hline S1 & 55 & 4 & 4 & Genetic \\
S2 & 51 & 1 & 3 & Meningitis \\
S3 & 70 & $<2$ & 3 & Unknown sudden \\
S4 & 31 & 2 & 7 & Unknown progressive \\
S5 & 66 & $>6$ & 1 & Unknown progressive \\
S6 & 60 & 3 & 2 & Unknown progressive \\
S7 & 61 & 18 & 3 & Unknown \\
S8 & 52 & 1 & 4 & Meningitis \\
\hline
\end{tabular}

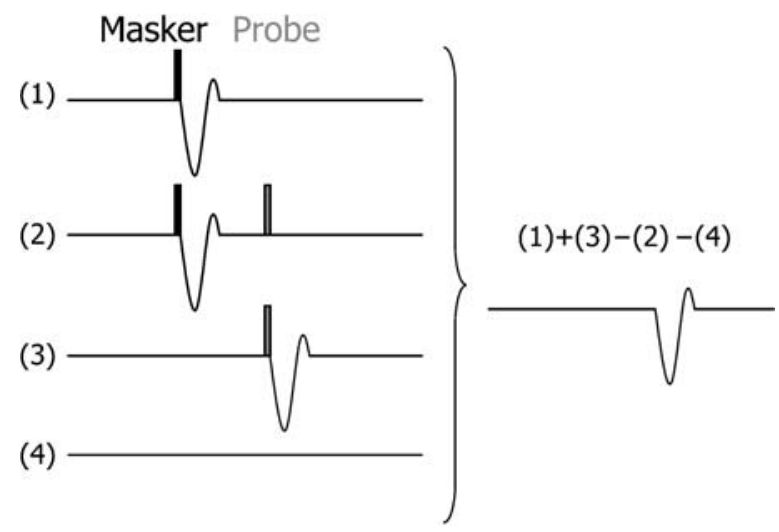

FIG. 1. Description of the masker-probe paradigm for artifact cancellation (Brown et al. 1990). Four traces are recorded: masker alone (1), masker and probe (2), probe alone (3), and zero stimulation (4) to correct for any DC offset. This method is based on the assumption that the nerve fibers are in their absolute refractory state when the probe is presented after the masker (2). The subtraction $(1)+(3)-(2)-(4)$ therefore yields the probe neural response with the artifact removed.

Research Ethics Committees and informed consent was obtained from all subjects.

Electrically evoked compound action potential recordings and psychophysical testing

Most contemporary CI systems allow the direct recording of the auditory nerve response, known as the electrically evoked compound action potential (ECAP). This is a noninvasive technique that consists of recording the intracochlear potential evolution after a stimulation pulse. To minimize electrical artifacts, we used the "masker-probe paradigm" (Brown et al. 1990), which relies on the fact that the neural response to a "probe" pulse is reduced by an immediately preceding "masker." By presenting the probe and masker either alone or in combination, it is possible to derive the neural response to the probe with the artifact "subtracted out" (cf. Fig. 1). In experiments 1 and 2, ECAPs were measured using several combinations of masker and probe. In all cases, masker and probe were presented in monopolar mode, on an electrode located in the middle of the array (electrode 9) with reference to the case (extracochlear) electrode of the implant. The responses were recorded using electrode 7 (approximately $2 \mathrm{~mm}$ apical to electrode 9) with reference to the ring ground electrode for S5 and the case electrode for all other subjects and were averaged over 128 sweeps. In experiment 3, psychophysical masking patterns were measured using the APEX experimental platform and the BEDCS software (cf. Macherey et al. 2006; Laneau et al. 2005). Masker and probe were also presented in monopolar mode, the masker on electrode 9 and the probe on an adjacent electrode (8 or 10$)$. 


\section{EXPERIMENTS}

Experiment 1: ECAP responses to anodic and cathodic phases

Rationale and methods. In experiment 1 , we recorded ECAPs from seven subjects $(\mathrm{S} 1-\mathrm{S} 7)$. The masker was a

A

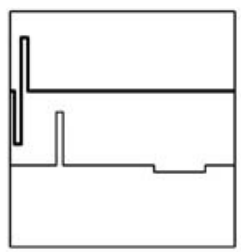

C Anodic phase responses
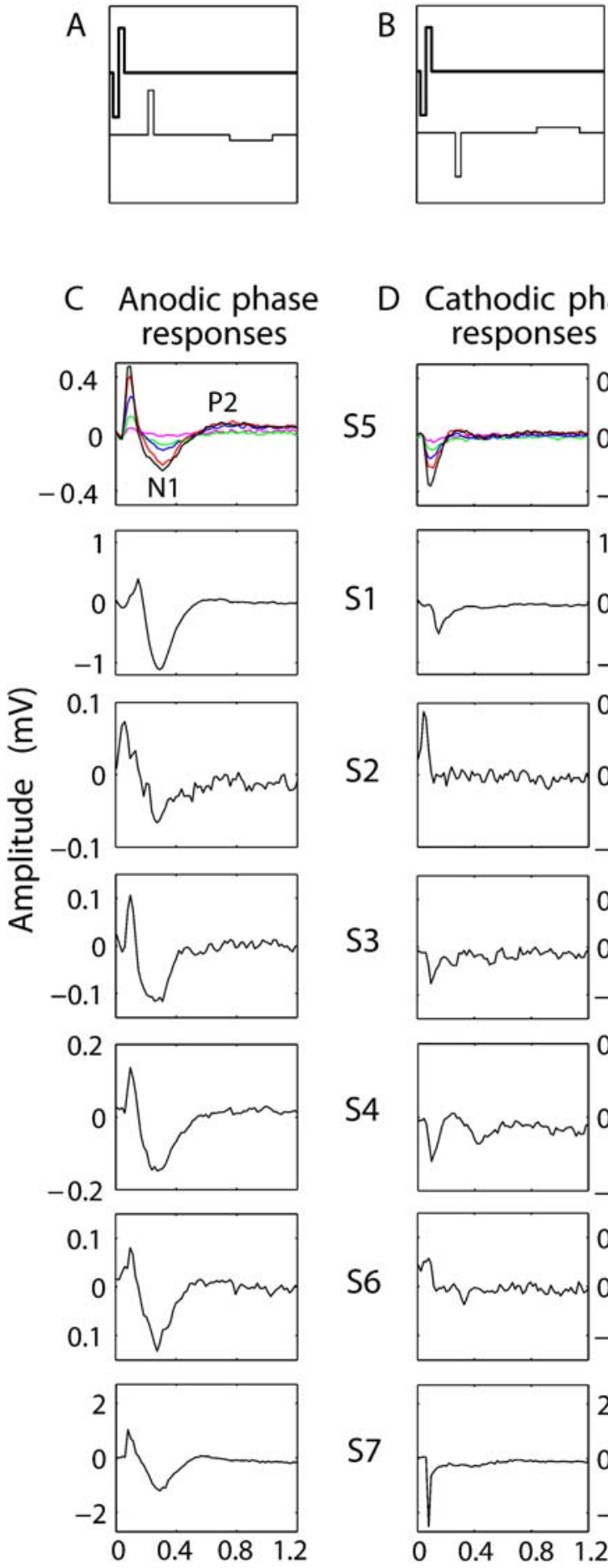

S5

D Cathodic phase responses

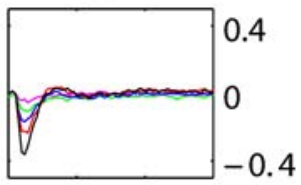

S1

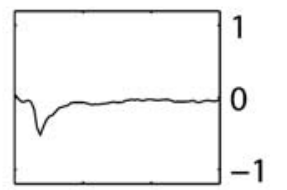

S2

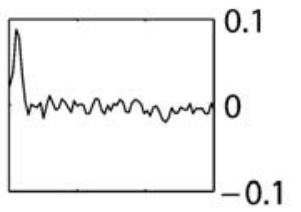

S3

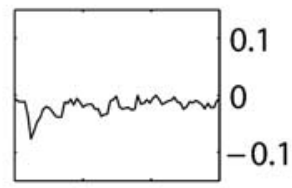

S4

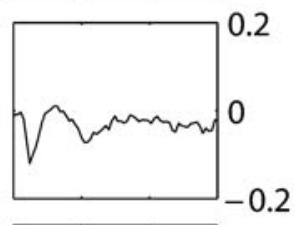

S6

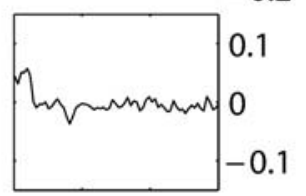

S7

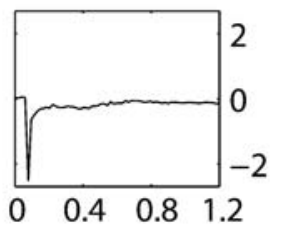

Time after probe onset (ms) cathodic-first, symmetric biphasic pulse with a $32-\mu \mathrm{s}$ phase duration presented at a comfortable level, determined subjectively for each subject before testing. The probes were either anodic-first (Fig. 2A) or cathodic-first (Fig. 2B) asymmetric waveforms with a phase separation of $2.9 \mathrm{~ms}$. The first (short) phase of the pulse was 32- $\mu$ s long, and the second (long) phase was eight times longer and lower in amplitude. The 2.9-ms phase separation present in the probe allowed us to measure the neural response to the first phase independently of the second. The two probes had the same level (between most comfortable and comfortably loud) and were each presented $500 \mu$ s after the end of the masker. Additionally, to differentiate neural responses from imperfectly cancelled stimulation artifacts, we recorded, in four of the subjects (S3S6), the responses to several probe levels, spanning the entire electrical dynamic range.

Results. An example of a level series is shown in the top panels of Figure 2C (anodic phase) and D (cathodic phase) for S5. The first narrow, short-latency peak observed for both anodic-first and cathodic-first probes almost certainly reflects a residual artifact because, for most subjects (with the exception of S2 and S6), its polarity is dependent on the probe polarity (positive and negative for anodic-first and cathodic-first probes, respectively), and its latency is similar for the two probes. For all subjects, the response to the anodic phase shows a biphasic shape with a first-negative peak $\mathrm{N} 1$ followed by a less robust positive peak P2. Only one (S4) of the seven subjects showed a measurable response to the cathodic phase, and only at the highest level of the dynamic range. For this subject, the latency of the response was longer for the cathodic-first $(420 \mu \mathrm{s})$ than for the anodic-first $(270 \mu \mathrm{s})$ probe, consistent with reports of animal auditory excitation (Miller et al. 1998, 1999a, 2004). Most of these animal data were, however, obtained using a recording electrode located at the nerve trunk, whereas our data reflect intracochlear recordings. The position of the recording electrode may have an important influence on the response latencies, and we will further consider

FIG. 2. ECAP responses to anodic and cathodic phases. A Masker (thick line) and probe (thin line) used to measure the anodic phase response. B Masker and probe used to measure the cathodic-phase response. C and D ECAPs evoked by the first phases of anodic-first (C) and cathodic-first (D) phase-separated asymmetric probes. The time reference corresponds to the onset of the probe. The second phase is not displayed as it occurs $2.9 \mathrm{~ms}$ after the first one. The top panels show an example of a level series obtained with subject S5. The different curves correspond to probe levels ranging from a soft $(320 \mu \mathrm{A})$ to a comfortably loud level $(576 \mu \mathrm{A})$ by steps of $64 \mu \mathrm{A}$, with the size of the fluctuations increasing monotonically with increasing current level. The other panels show the responses to the highest probe levels for all the other subjects. The N1 peak of the anodic response occurs, on average, $290 \mu$ s after the onset of the probe. 
this parameter in "Discussion." For now, it is worth noting that the major discrepancy with common animal data was that the amplitude of the response was, in our case, always greater for the anodic phase.

\section{Experiment 2: masking effectiveness of anodic} and cathodic phases-ECAP measures

Rationale and methods. The reliability of the maskerprobe paradigm strongly depends on the effectiveness of the masker, as the neural response obtained represents the subtraction of the masked response from the unmasked one. Instead of reflecting different neural responses, the anodic/cathodic amplitude difference obtained in experiment 1 may relate to the fact that the biphasic masker did not effectively mask the cathodic phase. This would be expected if, for example, the excitation sites for the biphasic masker and the cathodic probe were the central axon and the peripheral process, respectively. The peripheral process may, in such a case, not be driven in its refractory state by the masker, and the masker-probe paradigm would consequently subtract out the neural response. We performed, in experiment 2, another set of ECAP measures in six subjects (S1-S6) to verify that the anodic phase was also a more effective masker than the cathodic phase. Unlike the previous experiment, we introduced a 2.9-ms phase separation in the symmetric biphasic masker and assumed that the probe would be mainly masked by the masker's second phase (denoted as the masking phase). We recorded the responses to anodic-first and cathodic-first symmetric biphasic probes with no phase separation in the two conditions, "anodic masking" (Fig. 3A) and "cathodic masking" (Fig. 3B) phase, and also in a third "biphasic masking" (Fig. 3C) condition. This last condition, where the masker was a cathodic-first, symmetric biphasic pulse with no phase separation, was performed for two reasons. First, because this protocol is routinely used in clinics, the morphology of the resulting ECAP response is well documented. It usually

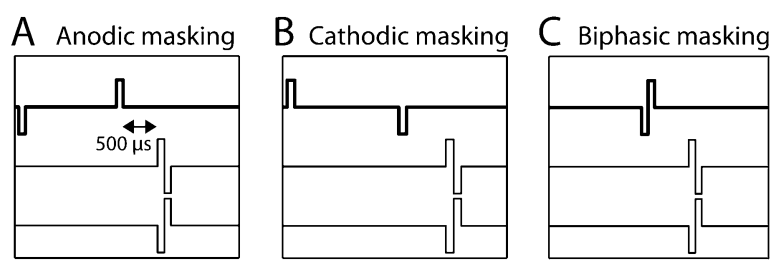

FIG. 3. Combinations of maskers (thick lines) and probes (thin lines) used in experiment 2. A Anodic masking condition. B Cathodic masking condition. C Biphasic masking condition. For each masking condition, two different symmetric biphasic probes were used: an anodic-first and a cathodic-first.

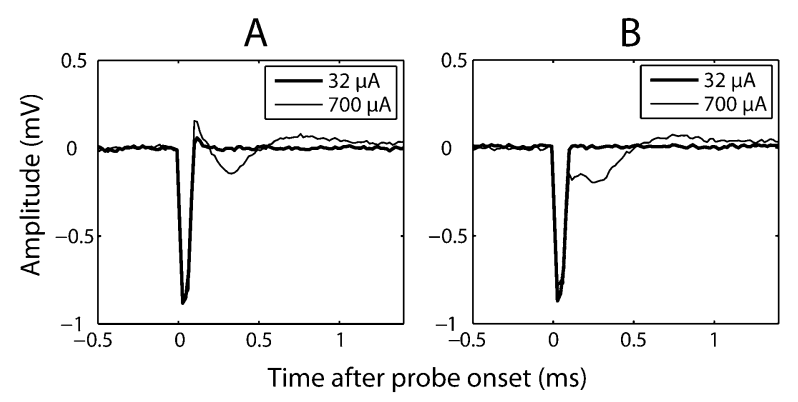

FIG. 4. Examples of uncancelled artifacts obtained in experiment 2 for S5. ECAP responses to cathodic-first (A) and anodic-first (B) biphasic probes in the anodic-masking condition at subthreshold (thick line) and suprathreshold (thin line) levels.

consists of an N1-P2 complex (Brown et al. 1990), similar to the response obtained in experiment 1 for the anodic phase. Second, it allowed us to compare the latencies of the probes' responses. If the anodic phase is the most excitatory phase of a biphasic pulse, we would expect the response to show a longer latency when the anodic phase is the second phase of the probe than when it is the first.

Results. For all masking conditions, a large uncancelled artifact was present at the probe onsets. Figure 4 shows examples of these artifacts obtained with S5 using biphasic cathodic-first (A) and anodic-first (B) probes in the anodic masking condition. The size of this artifact did not depend on the probe level and was still present at subthreshold levels (thick lines of Fig. 4). For means of clarity and because this uncancelled artifact was clearly distinct from the neural response, subsequent figures will only show the neural response part of the recordings. Note that an advantage of the present approach is that it compares the effects of different maskers, whereas the artifact occurred near the onset of the probe.

Figure 5A shows the anodic-first (thick line) and cathodic-first (thin line) probe responses in the biphasic masking condition for S1. The response latency of the N1 peak was shorter for the anodic-first probe, consistent with the neural activation being elicited primarily by the anodic phase. All subjects demonstrated this trend with a latency difference ranging from 0 to $80 \mu$ s (Fig. $5 \mathrm{~B}$ ), opposite to results obtained in the guinea pig (Klop et al. 2004). A $t$ test performed on the data from the six subjects revealed that this difference was significant $(d f=5, p=0.011)$. Apart from this latency difference, the responses to anodic-first and cathodic-first probes had similar amplitudes. To further improve artifact cancellation, the two responses were averaged for data analysis.

An example of the averaged responses of $\mathrm{S} 1$ is illustrated in Figure 5 for anodic masking $(\mathrm{C})$, cathodic masking (D), and biphasic masking (E). For the six subjects tested, the amplitude of the response (differ- 

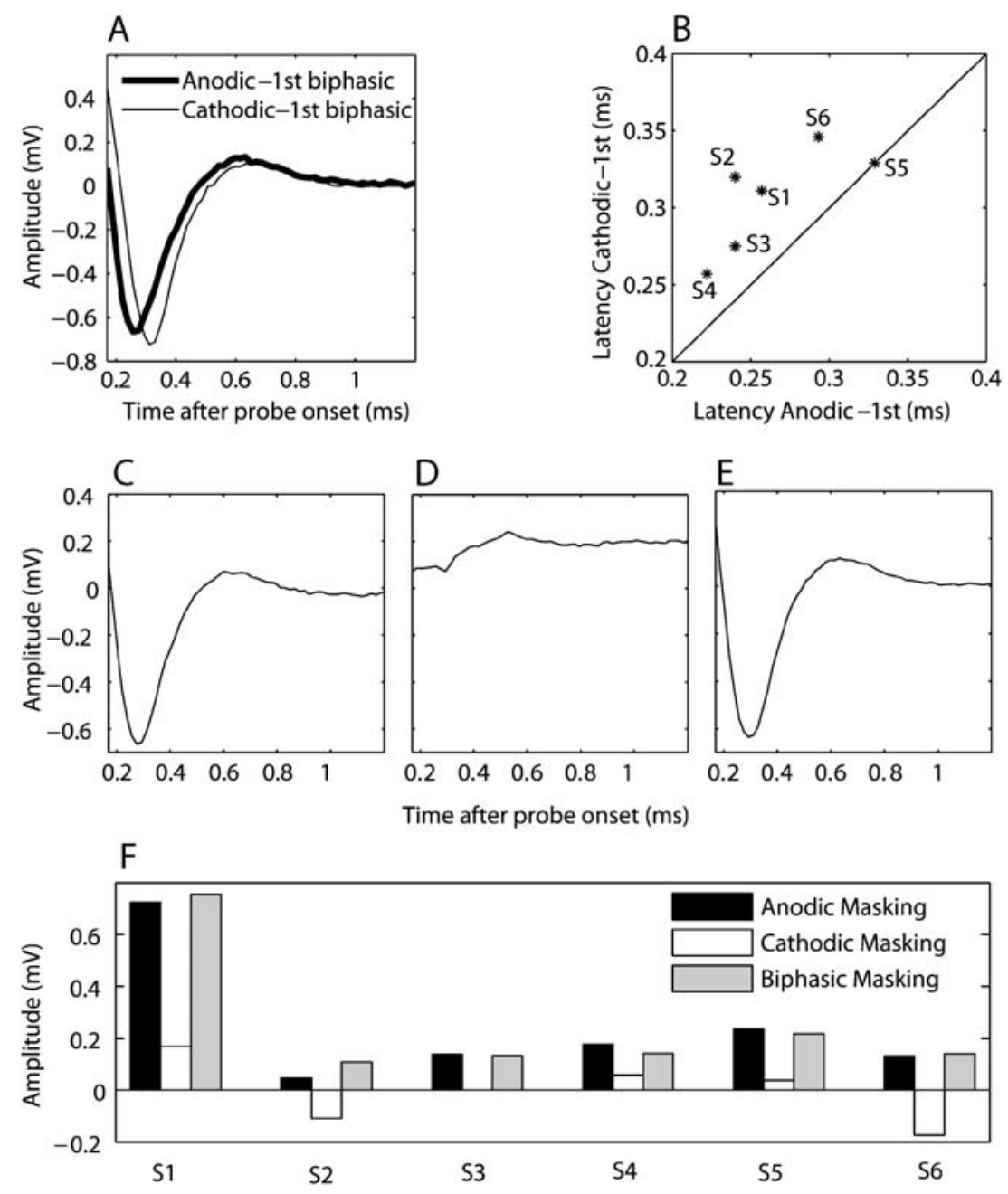

FIG. 5. Masking effectiveness of anodic and cathodic phases. A Example of ECAP recordings obtained with subject S1 for an anodicfirst (thick line) and a cathodic-first (thin line) biphasic probe in the biphasic masking condition. The response latency is shorter for the anodic-first probe. B Latency of the cathodic-first probe response vs. latency of the anodic-first probe response. For each subject, the two probes were presented at an identical level (comfortably loud) and also at the same level as the biphasic masker. $\mathbf{C}$, D, and $\mathbf{E}$ Example of ECAPs evoked by a symmetric biphasic probe with no phase separation in the three masker conditions: anodic masking $(\mathbf{C})$, cathodic masking $(\mathbf{D})$, and

ence between $\mathrm{N} 1$ and $\mathrm{P} 2$ ) was greater when the masking phase was anodic than when it was cathodic (Fig. 5F). This confirmed the previous finding that the anodic phase is more effective in driving auditory nerve fibers in their refractory state. Furthermore, the probe responses in the anodic masking and biphasic masking conditions were strikingly similar, suggesting that the anodic phase is the effective phase of a symmetric biphasic pulse. This last observation also argues strongly against the responses being influenced by an uncancelled masker artifact. Note that, although the anodic and cathodic maskers had the same level, and differed only in their leading polarity, the level of biphasic masking (E) for subject S1. Each trace corresponds to the average of the anodic-first and cathodic-first biphasic probe responses. In the cathodic masking condition, the neural response was sometimes not clearly measurable. In $\mathbf{D}$, for example, there is no excursion of the trace in the negative portion of the graph. Nevertheless, the recording still shows a voltage increase in the same region where the probe neural response occurs. The amplitude of the probe response was, in this case, assumed to be the magnitude of this increase. F Summary of ECAP amplitudes obtained in the anodic-masking (black bars), cathodicmasking (white bars), and biphasic-masking (gray bars) conditions.

the biphasic masker was presented, on average, $2.4 \mathrm{~dB}$ higher to have a loudness similar to that of the other two maskers. This increase in level reflects the influence of phase separation on loudness reported in previous publications (McKay and Henshall 2003; Carlyon et al. 2005; van Wieringen et al. 2005; Macherey et al. 2006). For S2 and S6, the amplitude is negative in the cathodic masking case because the subjects exhibited an inverted response (with a positive peak). This may mean that the cathodic phase acts as a facilitator and not as a masker for these specific subjects, possibly because of residual potential summation (Miller et al. 1997; De Balthasar et al. 2003). 
Experiment 3: masking effectiveness of anodic and cathodic phases-psychophysical measures

Rationale and methods. Because ECAPs in CIs can only be measured intracochlearly and not directly on the nerve trunk, the recordings potentially contain the neural response traveling not only toward the central auditory system (orthodromically) but also in the opposite direction, toward the fibers' periphery (antidromically; Miller et al. 2004). It is possible that this phenomenon occurred and that the larger ECAP amplitude of the anodic response obtained in experiment 1 did not reflect a larger response propagating toward the central auditory system (Cartee et al. 2005). Although the results of experiment 2 argue against this hypothesis, a perhaps more direct way to tease out this effect is to perform psychophysical measures, which require perceptual judgments from the subjects, thereby reflecting neural responses propagating centrally. The aim of experiment 3 was to compare the masking effectiveness of each polarity phase psychophysically and also to measure the most effective phase of an asymmetric pulse: Is it the short, high-amplitude or the long, low-amplitude one? We used two asymmetric pulse-train stimuli (anodic- and cathodic-first) with a 6.4-ms phase separation as maskers of a biphasic pulse-train probe presented $1 \mathrm{~ms}$ either after the first or after the second phase of the masker (Fig. 6A). Contrary to experiments 1 and 2 where we were constrained by the limited duration of the recording window, we used a longer phase separation to minimize refractory effects of one phase of the masker over the other. The masker was a 76pulse per second pulse train, either anodic-first (conditions $1 \mathrm{a}$ and $\mathrm{b}$ ) or cathodic-first (conditions $2 \mathrm{a}$ and $\mathrm{b}$ ) with a short phase of $22 \mu$ s and a long phase eight times longer and lower in amplitude. Masker and probe had a total duration of $400 \mathrm{~ms}$ and were temporally interleaved, the masker being presented on electrode 9 and the probe on electrode 8 , or, in an additional set of measures, on electrode 10. Four subjects (S1-S3 and S8) were asked to detect the anodic-first biphasic probe in the presence of one of the two maskers, in a three-interval, two-alternative forced-choice task where only one interval contained the probe. Feedback was provided and the level of the probe was adaptively varied in a two-down, one-up adaptive procedure that stopped after eight reversals. For subjects S1-S3, the step size was $32 \mu \mathrm{A}$ for the first two and $4 \mu \mathrm{A}$ for the last six reversals. For subject S8, the phase duration of the biphasic probe had to be increased to $43 \mu$ s to be able to reach a comfortably loud level, and the step sizes were 64 and $8 \mu \mathrm{A}$, respectively, for the first two and the last six reversals. Each estimate was the average value of the last six reversals. We also performed the same four measures with the polarity of the probe reversed (cathodic-first instead of anodic-first). We did this because, in a previous study (Macherey et al. 2006), we reported the presence of a low-amplitude current flow between the two phases of stimuli with relatively long phase separations (similar to the separation used here). The polarity of this current flow is opposite to the polarity of the preceding stimulus phase and is due to the passive discharge of the implant's DC-blocking capacitors. This current may influence the detection of the probe by predepolarizing or prehyperpolarizing the neural membrane after each phase. The results obtained in this condition were similar to the ones
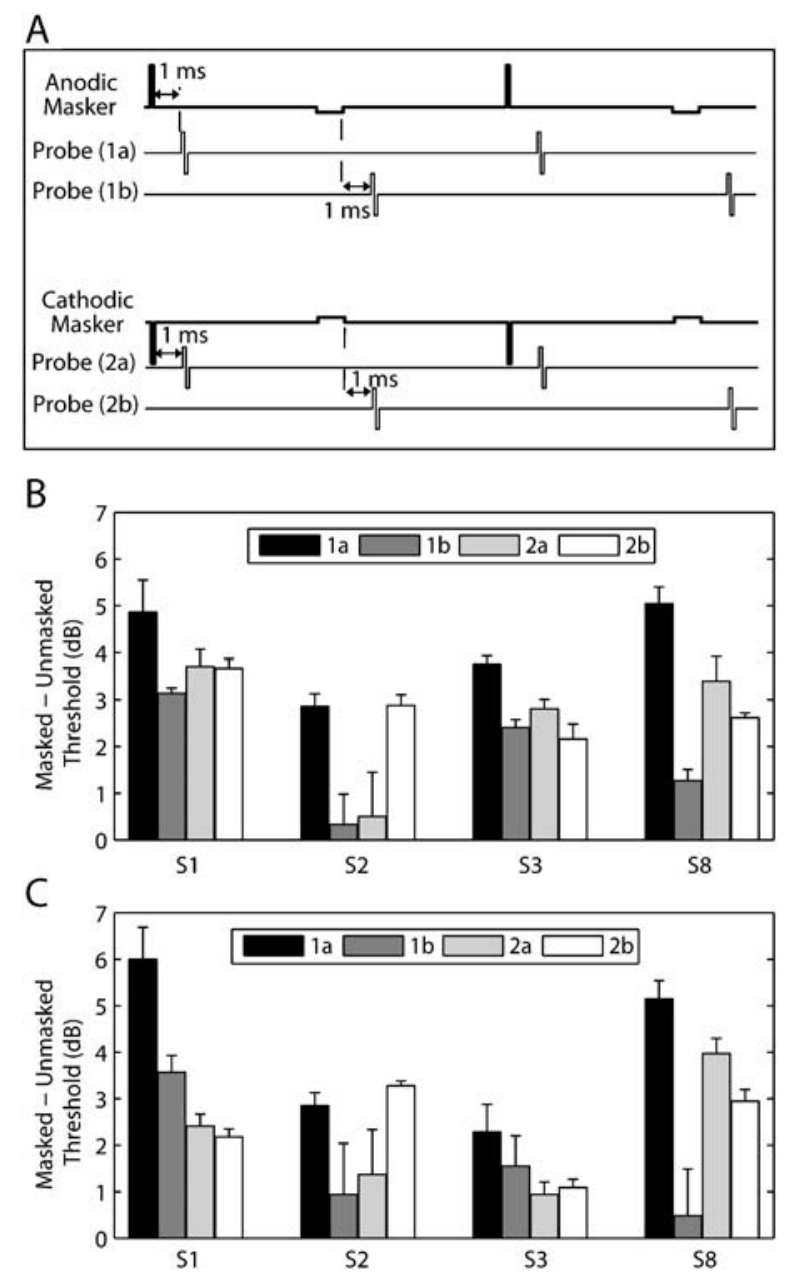

FIG. 6. Psychophysical masking patterns. A Description of the four experimental conditions. Anodic-first asymmetric masker (thick line) with a phase separation of $6.4 \mathrm{~ms}$ followed by a biphasic probe presented $1 \mathrm{~ms}$ after the end of the short, high-amplitude phase (1a) or $1 \mathrm{~ms}$ after the end of the long, low-amplitude phase $(1 \mathrm{~b})$ of the masker. Conditions $2 a$ and $2 b$ are identical to $1 a$ and $1 b$ except that the masker is reversed in polarity. B Difference between masked and unmasked threshold for the four conditions (1a, 1b, 2a, and 2b) and the four subjects ( $\mathrm{S} 1-\mathrm{S} 3$ and $\mathrm{S} 8$ ) for a probe on electrode 8 . The probe dynamic ranges were $6.5,4.2,3.9$, and $11.1 \mathrm{~dB}$ for $\mathrm{S} 1, \mathrm{~S} 2, \mathrm{~S} 3$, and S11, respectively. C Same as B with the probe on electrode 10. 
obtained with the initial stimulus polarity and will therefore not be shown here.

Results. The four CI subjects (S1-S3, S8) were first asked to balance in loudness the two opposite-polarity maskers at their MCL following a procedure described in Macherey et al. (2006). On average, the level of the cathodic masker was adjusted 1.6-dB higher than the anodic one, consistent with the anodic-first pulse being more effective than the cathodic-first one. These levels were used in the following part of the experiment where the subjects had to detect the presence of the biphasic probe in each of the four conditions and also in the unmasked case. Figure $6 \mathrm{~B}$ shows the threshold shifts (difference between masked and unmasked thresholds) obtained with the probe on electrode 8. For an anodic masker, the short, highamplitude (anodic) phase produced more masking than the long, low-amplitude (cathodic) phase for all subjects (conditions la vs. 1b). No clear pattern was, however, obtained in the case of a cathodic masker (2a vs. 2b), showing across-subject variability. More interestingly, although presented at a lower level, the short, high-amplitude anodic phase (1a) was a more effective masker than the short, high-amplitude cathodic phase (2a). Similar results were obtained with the probe on electrode 10 (Fig. 6C). These observations were corroborated by a two-way repeated measures ANOVA performed on the mean data of all subjects. The treatment factors were the probe channel ( 8 or 10$)$ and the probe condition. First, there was a significant effect of the probe condition $(F(3,9)=5.01, p=0.026)$ but no effect of the probe channel $(F(1,3)=0.52, p=$ $0.52)$ or of the interaction factor $(F(2.91,8.73)=0.22$, $p=0.88$ ). Second, pairwise comparisons revealed that the short, high-amplitude anodic phase was a better masker than the long, low-amplitude cathodic phase $(1 \mathrm{a}>1 \mathrm{~b}, p=0.037)$ and also than the short, highamplitude cathodic phase $(1 \mathrm{a}>2 \mathrm{a}, p=0.008)$. No significant difference was, however, found between the short cathodic and the long anodic phases $(p>$ 0.4). These results demonstrate that the larger ECAP response previously measured for anodic stimulation was not solely caused by antidromic propagation and strengthens the finding that auditory nerve fibers are more sensitive to anodic stimuli.

\section{DISCUSSION}

\section{Main findings}

We have reported the results of three electrophysiological and psychophysical masking experiments designed to compare the responses of the human auditory nerve to anodic and cathodic current flows. In experiment 1 , the anodic phase produced the usual N1-P2 ECAP morphology for all subjects, whereas the cathodic phase showed no measurable response except for one subject. This observation led us to conclude that the anodic phase was more effective than the cathodic phase. There are, however, at least three alternative explanations for this trend.

First, the cathodic phase response of experiment 1 may have had a shorter latency than the anodic phase response and may have consequently been mixed within the uncancelled stimulus artifact. Although this hypothesis is not supported by animal data, usually showing longer latencies for cathodic stimuli (Miller et al. 1998, 1999a, 2004), a potentially important confounding variable is the location of the recording electrode. Most animal ECAP data are recorded using an electrode positioned on the nerve trunk. Consequently, the shorter the response latency, the more central the excitation site is. This may, however, not be the case for intracochlear recordings where the recording and the stimulating electrodes are close to each other (Miller et al. 2004). Here, we used a recording electrode $2 \mathrm{~mm}$ apical to the stimulating one, similar to what is used clinically, and this choice may have affected the morphology and the latency of the ECAP responses. We note that our second experiment controlled for any possible effects of response latency to the probe by studying the effects of masker polarity for a fixed probe and by showing that, although the leading polarity of a biphasic probe did affect the response latency, it was always the case that responses were larger with an anodic than with a cathodic masker.

Second, as previously stated, the response to the anodic phase obtained in experiment 1 may not only represent the neural response traveling toward the central auditory system. As the recording electrode is located at the periphery of the nerve fibers, the recorded response possibly contains the antidromic response of the fibers. Although this is possible, this would necessarily imply that the nerve fibers still have remaining peripheral processes to allow the propagation of antidromic spikes. In such a case, this phenomenon may not occur in subjects with a long duration of deafness and presumably a poor peripheral processes' survival. Furthermore, the psychophysical report of experiment 3 demonstrates that the orthodromic response (propagating centrally) is also larger for the anodic than for the cathodic phase. Antidromic propagation cannot therefore be the only account for our finding.

Third, the cathodic phase may excite a different subpopulation of nerve fibers than a biphasic pulse with no phase separation. In such a case, the maskerprobe paradigm that we used in Experiments 1 and 2 would not be effective in the measure of the cathodic phase response, i.e., if the masker does not effectively mask the probe, the subtraction method would not 
allow the neural response to appear. Although the present data cannot rule out this possibility, it is worth noting that the masker-probe paradigm was successfully used in the measure of the anodic phase response. This would mean that a biphasic pulse (whether anodic- or cathodic-first) with no phase separation excites the same subpopulation as a single anodic phase but a different subpopulation than a single cathodic phase. Consequently, this would not change the conclusion that the anodic phase is the most "effective" phase of a biphasic pulse and that the ECAPs recorded clinically most likely represent the response to the anodic phase of biphasic pulses.

Although the observation of a higher sensitivity to anodic current flows is surprising, some animal data have already shown such a result. Miller et al. (1998) recorded ECAPs to monophasic anodic and cathodic pulses from the cat and the guinea pig. They observed lower cathodic thresholds for cats but lower anodic thresholds for guinea pigs. The reason for this species' discrepancy was, however, unclear, and the authors proposed that it may relate to differences in anatomy or in electrode size and position. Other stimulus parameters have shown to affect human and animal subjects in different ways (Shepherd and Javel 1999; Carlyon et al. 2005), and several factors may underlie those species' differences, including differences in anatomy, neural properties, neural survival, and electrode geometry (reviewed by Miller et al. 1999b).

\section{Factors affecting polarity sensitivity}

The simplest model of extracellular stimulation that can account for a lower cathodic threshold is the activating function of Rattay (1989). This model describes the spatial pattern of polarization of an infinite, homogeneous fiber subjected to an extracellular electrical current. A cathodic stimulus produces a large depolarization of the fiber proximal to the electrode and a smaller hyperpolarization at both sides of the depolarized region, whereas an anodic stimulus produces the exact opposite pattern of polarization. This model is, however, too restrictive in the case of auditory nerve fibers, which are made of several excitable elements (peripheral process, unmyelinated cell body, and central axon) that presumably have different biophysical properties and, hence, different activation thresholds. Moreover, auditory nerve fibers are curved, and the spatial pattern of polarization produced by an electrical pulse may show multiple regions of depolarization and hyperpolarization (Rattay 1999; Rattay et al. 2001a). There are at least three factors that may influence polarity sensitivity: the electrode position, the nerve parameters, and the stimulus current level. They are discussed in the following paragraphs.
Ranck (1975) reviewed the different mechanisms of electrical excitation of the central nervous system and showed that the electrode position relative to the fiber had a crucial effect on its sensitivity to polarity. He reported that myelinated fibers were more easily excited by cathodic stimuli when the electrode was placed over the central axon. However, for an electrode located near the cell body, on the opposite side from the central axon, the lowest threshold was often obtained with an anodic stimulus. Rattay (1999) and McIntyre and Grill (1999) found similar placedependent polarity effects on their modeled central neurons. In another modeling study, Rubinstein (1993) focused on the excitation process near the axon termination. He also demonstrated the extreme dependence of polarity sensitivity on the exact electrode position. As a CI electrode presumably excites a large number of nerve fibers with different orientations and locations, it is possible that each fiber is excited differently by anodic and cathodic phases (Rattay et al. 2001b). The present study was restricted to electrodes mid-way along the electrode array. Subsequent studies should explore other electrode positions and other electrode designs.

Computational models have provided a simple way to investigate the influence of neural parameters. In two different modeling studies, Rattay et al. (1999, 2001a) suggested that intact human auditory nerve fibers would be more sensitive (showing lower excitation thresholds) to cathodic stimuli, whereas fibers with degenerated or demyelinated peripheral processes would be more sensitive to anodic stimuli. First, they predicted that, for intact fibers, the depolarization magnitude would be maximal at the level of the peripheral process and would be larger for a cathodic than for an anodic pulse. Second, for fibers without peripheral processes, their model showed that the only site that could be depolarized by a cathodic pulse was the cell body, whereas an anodic pulse could depolarize the central axon. Given that the cell body was modeled using a channel density ten times smaller than for the central axon, it had a much higher threshold than the central axon, thereby leading to a lower anodic threshold. A similar effect occurred in the case of demyelination of the peripheral process because unmyelinated fibers need much stronger currents to be excited than myelinated ones. Given that peripheral processes in deafened ears are believed to be somewhat degenerated (Fayad and Linthicum 2006) and that this degeneration is often accompanied by demyelination, the predictions of Rattay et al. (1999, 2001a) give a plausible explanation for the higher sensitivity to anodic stimulus currents observed in implanted subjects. This would therefore imply that the excitation site is at the level of the central axon, which is consistent with a report of van 
den Honert and Stypulkowski (1984) on the responses of cats' fibers with and without peripheral processes and cell bodies. They found the responses of the degenerated fibers to closely resemble those of the normal fibers stimulated at high current levels. Although the exact location of the excitation site is still a matter of debate and probably depends on the electrode configuration and on the stimulus current level, Miller et al. (1999a) demonstrated that cats' fibers were almost always excited at the level of their central axon with monopolar stimulation. The response latencies obtained in experiment 1 for anodic stimulation (290 $\mu$ s on average) also argue for a stimulation at the level of the central axon, given that they are similar to the latencies commonly observed in human CI subjects for symmetric biphasic waveforms but also comparable to those measured intracochlearly in cats for anodic pulses (Brown et al. 1990; Miller et al. 2004). Given that the main morphological discrepancies between humans and cats are the length of the peripheral processes (longer in humans) and the size and degree of myelination of the spiral ganglion cells (larger and unmyelinated in humans; Liberman and Oliver 1984; Spoendlin and Schrott 1989; Rattay et al. 2001a), we would expect significant ECAP latency differences if the auditory nerve fibers were excited at their peripheral process or cell body sites but not if they were excited at the level of their central axon. To test whether neural degeneration and demyelination are the causes of our finding, additional psychophysical measures may be performed. Specifically, in ears with substantial degeneration, the dominance of the anodic phase should be enhanced, the effect of phase separation on threshold should be reduced (Prado-Guitierrez et al. 2006), and the slopes of the strength-duration functions should be shallower (Miller et al. 1995).

Finally, we reported in a previous study (Macherey et al. 2006) a comparison of thresholds and MCLs for anodic- and cathodic-first asymmetric pulses. Although we did find lower MCLs for anodic-first pulsesconsistent with the present results-we did not observe any difference at threshold. It is possible that the higher sensitivity to anodic stimuli is specific to suprathreshold levels. One hypothesis proposed by Macherey et al. (2006) was that cathodic-first asymmetric pulses may produce a cathodal block at high levels. This seems, however, unlikely given that cathodal blocking should occur at very high levels above threshold (approximately eight times higher; Ranck 1975). This would correspond to a current level 18-dB higher than threshold, therefore exceeding the common dynamic range values of CI users (for example, the probe dynamic ranges of experiment 3 ranged from about 4 to $11 \mathrm{~dB}$ ). Another possibility is that the cluster of fibers recruited at threshold is equally sensitive to anodic and cathodic phases or that, alternatively, approximately the same number of fibers is excited by an anodic and by a cathodic phase. At higher levels, more spatially remote fibers may be recruited, and it is possible that a majority of these fibers are more sensitive to anodic stimulation. Interestingly, in their spatial modeling study of the human cochlea, Rattay et al. (2001b) predicted that the nerve fibers close to the stimulating electrode would demonstrate lower cathodic than anodic thresholds but that fibers more spatially remote would in some cases exhibit the opposite trend. However, we note that in experiment 3 , the greater masking effectiveness of the anodic phase was observed for probes presented to electrodes adjacent to that used for the masker.

\section{Applications to Cls}

Our results have implications not only for theories of electrical stimulation of the human auditory nerve but also for the development of future CIs. Asymmetric pulses may be used in a CI speech processing strategy for two reasons: to reduce power consumption or to increase spatial selectivity.

First, we previously suggested that asymmetric pulses presented in monopolar mode would achieve some worthwhile power savings (Macherey et al. 2006). If such pulses were implemented in a speech processing strategy, then, the results of experiment 3 suggest that the short, high-amplitude phase of the pulses should be anodic relative to the active electrode. In this case, the effect of the long, low-amplitude phase on neural excitation should be kept minimal.

Second, for so-called longitudinal bipolar stimulation, where each intracochlear electrode is stimulated with reference to another nearby intracochlear electrode, the pattern of excitation along the cochlea can sometimes show a bimodal shape with peaks of excitation near the two stimulating electrodes (Kral et al. 1998; Chatterjee et al. 2006). Frijns et al. (1996) suggested that this bimodality may be reduced by the use of asymmetric pulses. Their computational model showed that the nerve fibers proximal to the electrode for which the short, high-amplitude phase is cathodic would be more effectively excited than the fibers proximal to the other electrode. Our results suggest that a similar reduced bimodality and enhanced spatial selectivity may be obtained in CIs, but, crucially, with more excitation near the electrode presenting the short, anodic phase. Note that, in the present work, we used asymmetric pulses with relatively long phase separations, which are unlikely to be useful in multichannel CIs because they would require an extremely low repetition rate. However, we would expect this enhanced spatial selectivity to also hold for bipolar asymmetric stimuli with a shorter or no phase separation, which may be more appropriate 
candidates for implementation in a CI speech processing strategy.

\section{ACKNOWLEDGMENT}

We thank C. A. Miller for comments on an earlier version of the manuscript. This work was supported by the Fonds voor Wetenschappelijk Onderzoek FWO-Vlaanderen (FWO G.0233.01) and the Research Council of the Katholieke Universiteit Leuven (OT/03/58).

OPEN ACCESS This article is distributed under the terms of the Creative Commons Attribution Noncommercial License which permits any noncommercial use, distribution and reproduction in any medium, provided the original author(s) and source are credited.

\section{REFERENCES}

Brown CJ, Aвbas PJ, Gantz BJ. Electrically evoked whole-nerve action potentials: data from human cochlear implant users. J. Acoust. Soc. Am. 88:1385-1391, 1990.

Brummer SB, Turner MJ. Electrochemical considerations for safe electrical stimulation of the nervous system with platinum electrodes. IEEE Trans. Biomed. Eng. 24:59-63, 1977.

Carlyon RP, van Wieringen A, Deeks JM, Long CJ, Lyzenga J, WOUTERs J. Effect of inter-phase gap on the sensitivity of cochlear implant users to electrical stimulation. Hear. Res. 205:210-224, 2005.

Cartee LA, Finley CC, van den Honert C, Lawson DT, Wilson BS. Intracochlear evoked potential responses to biphasic pulses with an interpulse gap. Asilomar Conference on Implantable Auditory Prostheses, Pacific Grove, USA, p 84, 2005.

Chatterjee M, Galvin JJ III, Fu QJ, Shannon RV. Effects of stimulation mode, level and location on forward-masked excitation patterns in cochlear implant patients. J. Assoc. Res. Otolaryngol. 7:15-25, 2006.

De Balthasar C, Boex C, Cosendai G, Valentini G, Sigrist A, Pelizzone M. Channel interactions with high-rate biphasic electrical stimulation in cochlear implant subjects. Hear. Res. 182:77-87, 2003.

Fayad JN, Linthicum FH, JR. Multichannel cochlear implants: relation of histopathology to performance. Laryngoscope 116:1310-1320, 2006.

Frijns JHM, de Snoo SL, ten Kate JH. Spatial selectivity in a rotationally symmetric model of the electrically stimulated cochlea. Hear. Res. 95:33-48, 1996.

Hartmann R, Topp G, Kunke R. Discharge patterns of cat primary auditory fibers with electrical stimulation of the cochlea. Hear. Res. 13:47-62, 1984.

Klop WMC, Hartlooper A, Briaire JJ, Frijns JHM. A new method for dealing with the stimulus artifact in electrically evoked compound action potential measurements. Acta Otolaryngol. 124:137-143, 2004.

Kral A, Hartmann R, Mortazavi D, Klinke R. Spatial resolution of cochlear implants: the electrical field and excitation of auditory afferents. Hear. Res. 121:11-28, 1998.
Laneau J, Boets B, Moonen M, van Wieringen A, Wouters J. A flexible auditory research platform using acoustic and electric stimuli for adults and young children. J. Neurosci. Methods 142:131-136, 2005.

Liberman MC, Oliver ME. Morphometry of intracellularly labeled neurons in the auditory nerve: correlations with functional properties. J. Comp. Neurol. 223:163-176, 1984.

Macherey O, van Wieringen A, Carlyon RP, Deeks JM, Wouters J. Asymmetric pulses in cochlear implants: effects of pulse shape, polarity and rate. J. Assoc. Res. Otolaryngol. 7:253266, 2006.

MCINTYRe CC, Grill WM. Excitation of central nervous system neurons by nonuniform electric fields. Biophys. J. 76:878-888, 1999.

McKay CM, Henshall KR. The perceptual effects of interphase gap duration in cochlear implant stimulation. Hear. Res. 181:94-99, 2003.

Miller CA, Faulkner MJ, Pfingst Be. Functional responses from guinea pigs with cochlear implants. II. Changes in electrophysiological and psychophysical measures over time. Hear. Res. 92:100-111, 1995.

Miller AL, Morris DJ, Pfingst BE. Interactions between pulse separation and pulse polarity order in cochlear implants. Hear. Res. 109:21-33, 1997.

Miller CA, Abbas PJ, Rubinstein JT, Robinson BK, Matsuoka AJ, WOODWORTH G. Electrically evoked compound action potentials of guinea pig and cat: responses to monopolar, monophasic stimulation. Hear. Res. 119:142-154, 1998.

Miller CA, Abbas PJ, Robinson BK, Rubinstein JT, Matsuoka AJ. Electrically evoked single-fiber action potentials from cat: responses to monopolar, monophasic stimulation. Hear. Res. 130:197-218, 1999a.

Miller AL, Smith DW, Pfingst BE. Across-species comparisons of psychophysical detection thresholds for electrical stimulation of the cochlea: I. Sinusoidal stimuli. Hear. Res. 134:89-104, 1999b.

Miller CA, Abbas PJ, Robinson BK. Response properties of the refractory auditory nerve fiber. J. Assoc. Res. Otolaryngol. 2:216232, 2001.

Miller CA, Abbas PJ, Hay-McCutcheon MJ, Robinson BK, Nourski KV, JENG FC. Intracochlear and extracochlear ECAPs suggest antidromic action potentials. Hear. Res. 198:75-86, 2004.

Moon AK, Zwolan TA, Pfingst BE. Effects of phase duration on detection of electrical stimulation of the human cochlea. Hear. Res. 67:166-178, 1993.

Morsnowski A, Charasse B, Collet L, Killian M, Müller-Deile J. Measuring the refractoriness of the electrically stimulated auditory nerve. Audiol. Neurootol. 11:389-402, 2006.

Prado-Guttierrez P, Fewster LM, Heasman JM, McKay CM, Shepherd RK. Effect of interphase gap and pulse duration on electrically evoked potentials is correlated with auditory nerve survival. Hear. Res. 215:47-55, 2006.

RANCK JB, JR. Which elements are excited in electrical stimulation of mammalian central nervous system: a review. Brain Res. 98:417440, 1975.

RatTAY F. Analysis of models for extracellular fiber stimulation. IEEE Trans. Biomed. Eng. 36:676-682, 1989.

RatTaY F. The basic mechanism for the electrical stimulation of the nervous system. Neuroscience 89:335-346, 1999.

Rattay F, Lutter P, Felix H. A model of the electrically excited human cochlear neuron. I. Contribution of neural substructures to the generation and propagation of spikes. Hear. Res. 153:4363, 2001a.

Rattay F, Leao RN, Felix H. A model of the electrically excited human cochlear neuron. II. Influence of the three-dimensional 
cochlear structure on neural excitability. Hear. Res. 153:64-79, 2001b.

Rubinstein JT. Axon termination conditions for electrical stimulation. IEEE Trans. Biomed. Eng. 40:654-663, 1993.

SHEPHERD RK, JAVEL E. Electrical stimulation of the auditory nerve: II. Effect of stimulus waveshape on single fibre response properties. Hear. Res. 130:171-188, 1999.

Spoendlin H, Schrott A. Analysis of the human auditory nerve. Hear. Res. 43:25-38, 1989.
Stypulkowski PH, van den Honert C. Physiological properties of the electrically stimulated auditory nerve. I. Compound action potential recordings. Hear. Res. 14:205-223, 1984.

van Wieringen A, Carlyon RP, Laneau J, Wouters J. Effects of waveform shape on human sensitivity to electrical stimulation of the inner ear. Hear. Res. 200:73-86, 2005.

van den Honert C, Stypulkowski PH. Physiological properties of the electrically stimulated auditory nerve. II. Single fiber recordings. Hear Res. 14:225-243, 1984. 